\title{
Note about Hamiltonian formalism of healthy extended Hořava-Lifshitz gravity
}

\author{
Josef Klusoň \\ Department of Theoretical Physics and Astrophysics, \\ Faculty of Science, Masaryk University, \\ Kotlářská 2, 611 3\%, Brno, Czech Republic \\ Theory Division, Physics Department, CERN, \\ CH-1211 Geneva 23, Switzerland \\ E-mail: klu@physics.muni.cz
}

ABSTRACT: In this paper we continue the study of the Hamiltonian formalism of the healthy extended Hořava-Lifshitz gravity. We find the constraint structure of given theory and argue that this is the theory with the second class constraints. Then we discuss physical consequence of this result. We also apply the Batalin-Tyutin formalism of the conversion of the system with the second class constraints to the system with the first class constraints to the case of the healthy extended Hořava-Lifshitz theory. As a result we find new theory of gravity with structure that is different from the standard formulation of Hořava-Lifshitz gravity or General Relativity.

Keywords: Classical Theories of Gravity, Models of Quantum Gravity

ArXiv EPRINT: 1004.3428 


\section{Contents}

1 Introduction 1

2 Review of healthy extended Hořava-Lifshitz gravity 2

3 Batalin-Tyutin method for healthy extended Hořava-Lifshitz gravity 9

4 Conclusion $\quad 13$

\section{Introduction}

Last year Petr Hořava proposed new approach for the formulation of UV finite quantum theory of gravity [1-3]. The basic idea of this approach is to modify the UV behavior of the general theory so that the theory is perturbatively renormalizable. However this modification is only possible on condition when we abandon Lorentz symmetry in the high energy regime: in this context, the Lorentz symmetry is regarded as an approximate symmetry observed only at low energy.

Succeeding studies of the Hořava-Lifshitz gravity showed that in this model propagates an extra scalar mode with respect to General Relativity and appears to be burdened with serious shortcomings, such as instabilities, overconstrained evolution and strong coupling at low energies [5, 6, 27]. In [7] extension of Hořava-Lifshitz gravity was proposed, after noticing that terms involving $N$ and its spatial derivatives can be included in the potential term in the action without violating the symmetry of the action. The scalar mode in this model exhibits improved behavior $[8],{ }^{1}$ but see also [26].

Since the healthy extended Hořava-Lifshitz gravity is interesting proposal of alternative theory of gravity that contains spatial gradient of the lapse function we mean that it deserves to be studied from different points of view. We started the investigation of this theory in [9] where we discovered that the healthy extended Hořava-Lifshitz theory has very interesting Hamiltonian structure. We showed that due to the presence of the spatial derivatives of the lapse function in the Lagrangian the primary constraint $p_{N} \approx 0$ and corresponding secondary constraint are the second class constraints. This fact makes the theory completely different from the original Hořava-Lifshitz theory of gravity without projectability condition that seems to suffer from severe problems as was shown explicitly in $[10,12] .^{2}$ In particular, it was shown in [12] that the Hořava-Lifshitz gravity without the projectability condition has very peculiar property in the sense that the Hamiltonian

\footnotetext{
${ }^{1}$ It is important to stress that there exists also the second fundamental formulation of the Hořava-Lifshitz gravity where the lapse function $N$ depends on $t$ only. This version is known as Hořava-Lifshitz gravity with projectability condition. For review and extensive discussion of this version of theory, see [11].

${ }^{2}$ For an alternative approaches, see [28-30].
} 
constraints are the second class constraints and that the gravitational Hamiltonian vanishes strongly. On the other hand the healthy extended Hořava-Lifshitz gravities offers surprising resolution of this problem. Explicitly, since $p_{N}$ and corresponding secondary constraint are the second class constraints their can be explicitly solved. Then we can express $N$ as a function of canonical variables $g_{i j}, p^{i j}$, at least at principle. Further, the reduced phase space of healthy extended Hořava-Lifshitz theory is spanned by $g_{i j}, p^{i j}$. The important point is that the Hamiltonian constraint as we know from the General Relativity or from the healthy extended Hořava-Lifshitz gravity is absent. This remarkable observation implies that the healthy extended Hořava-Lifshitz gravities can provide solution of the problem of time in gravity [16]. In fact, according to standard analysis of the constraint systems all phase space functional should have weakly vanishing Poisson brackets with the constraints. In case of General Relativity the Hamiltonian is the linear combination of the constraints and hence any observable Poisson commutes with the Hamiltonian on the constraint surface and consequently any observable does not evolve with time. This serious problem of General Relativity was investigated in many papers in the past, see for example [20-24]. On the other hand the observable in the healthy extended Hořava-Lifshitz gravity is defined as phase-space functional that is invariant under spatial diffeomorphism. This is clearly much weaker condition than in General Relativity and hence it is possible to define observable in natural way with clear physical interpretation.

An important drawback of our analysis is that we will not able to solve explicitly the second class constraints and hence an explicit form of the Hamiltonian will not be found. In fact, since the potential $V$ given in [7] has complicated dependence on the metric $g_{i j}$ and the vector $a_{i}=\frac{\partial_{i} N}{N}$ the resulting Hamiltonian will be given as the sum of infinite terms with probably non-local dependence on $g_{i j}$ and $p^{i j}$.

For that reason we tried to implement the Batalin-Tyutin formalism [25] for the healthy extended Hořava-Lifshitz gravity in order to convert the second class constraints to the Poisson commuting first ones. As a result of this conversion we find the healthy extended Hořava-Lifshitz gravity where the extended phase space is spanned by dynamical variables $\left(g_{i j}, p^{i j}, N, p_{N}, N^{i}, p_{i}, \Phi^{1}, \Phi^{2}\right)$ where $\Phi^{1}, \Phi^{2}$ are new-dynamical fields that are necessary for this conversion. Unfortunately we will not be able to determine the Hamiltonian and all the first class constraints in the closed form.

The organization of this paper is as follows. In the next section we review the main properties of the healthy extended Hořava-Lifshitz gravity and perform its Hamiltonian formulation. Then in section (3) we review the main properties of the Batalin-Tyutin formalism and apply it for the healthy extended Hořava-Lifshitz theory. Finally in conclusion (4) we outline our results and suggest possible extension of this work.

\section{Review of healthy extended Hořava-Lifshitz gravity}

Let us consider $D+1$ dimensional manifold $\mathcal{M}$ with the coordinates $x^{\mu}, \mu=0, \ldots, D$ and where $x^{\mu}=(t, \mathbf{x}), \mathbf{x}=\left(x^{1}, \ldots, x^{D}\right)$. We presume that this space-time is endowed with the metric $\hat{g}_{\mu \nu}\left(x^{\rho}\right)$ with signature $(-,+, \ldots,+)$. Suppose that $\mathcal{M}$ can be foliated by a family of space-like surfaces $\Sigma_{t}$ defined by $t=x^{0}$. Let $g_{i j}, i, j=1, \ldots, D$ denotes the metric on $\Sigma_{t}$ 
with inverse $g^{i j}$ so that $g_{i j} g^{j k}=\delta_{i}^{k}$. We further introduce the operator $\nabla_{i}$ that is covariant derivative defined with the metric $g_{i j}$. We introduce the future-pointing unit normal vector $n^{\mu}$ to the surface $\Sigma_{t}$. In ADM variables we have $n^{0}=\sqrt{-\hat{g}^{00}}, n^{i}=-\hat{g}^{0 i} / \sqrt{-\hat{g}^{00}}$. We also define the lapse function $N=1 / \sqrt{-\hat{g}^{00}}$ and the shift function $N^{i}=-\hat{g}^{0 i} / \hat{g}^{00}$. In terms of these variables we write the components of the metric $\hat{g}_{\mu \nu}$ as

$$
\begin{array}{lll}
\hat{g}_{00}=-N^{2}+N_{i} g^{i j} N_{j}, & \hat{g}_{0 i}=N_{i}, & \hat{g}_{i j}=g_{i j}, \\
\hat{g}^{00}=-\frac{1}{N^{2}}, & \hat{g}^{0 i}=\frac{N^{i}}{N^{2}}, & \hat{g}^{i j}=g^{i j}-\frac{N^{i} N^{j}}{N^{2}} .
\end{array}
$$

Then it is easy to see that

$$
\sqrt{-\operatorname{det} \hat{g}}=N \sqrt{\operatorname{det} g} .
$$

The action of the healthy extended Hořava-Lifshitz theory takes the form

$$
S=\int d t d^{D} \mathbf{x} \sqrt{g} N\left(K_{i j} \mathcal{G}^{i j k l} K_{k l}-E^{i j} \mathcal{G}_{i j k l} E^{k l}-V\left(g_{i j}, a_{i}\right)\right),
$$

where we introduced the extrinsic derivative

$$
K_{i j}=\frac{1}{2 N}\left(\partial_{t} g_{i j}-\nabla_{i} N_{j}-\nabla_{j} N_{i}\right),
$$

and where the generalized metric $\mathcal{G}^{i j k l}$ is defined as

$$
\mathcal{G}^{i j k l}=\frac{1}{2}\left(g^{i k} g^{j l}+g^{i l} g^{j k}\right)-\lambda g^{i j} g^{k l},
$$

where $\lambda$ is real constant. Note that inverse $\mathcal{G}_{i j k l}$ is equal to

$$
\mathcal{G}_{i j k l}=\frac{1}{2}\left(g_{i k} g_{j l}+g_{i l} g_{j k}\right)-\tilde{\lambda} g_{i j} g_{k l},
$$

where $\tilde{\lambda}=\frac{\lambda}{D \lambda-1}$. Further, $E^{i j}$ are defined using the variation of $D$-dimensional action $W\left(g_{k l}\right)$

$$
\sqrt{g} E^{i j}=\frac{\delta W}{\delta g_{i j}} .
$$

These objects were introduced in the original work [1]. However it is possible to consider theory when $E^{i j} \mathcal{G}_{i j k l} E^{k l}$ is replaced with more general potential that is a function of $g_{i j}$ and their covariant derivatives. Further, the potential $V(a, g)$ depends on $g_{i j}$ and on $D$-dimensional vector $a_{i}$ constructed from the lapse function $N(t, \mathbf{x})$ as

$$
a_{i}=\frac{\partial_{i} N}{N} .
$$

It can be easily shown that the action (2.3) is invariant under foliation preserving diffeomorphism

$$
t^{\prime}-t=f(t), \quad x^{i}-x^{i}=\xi^{i}(t, \mathbf{x}),
$$

the lapse $N$, the shift $N^{i}$ and metric $g_{i j}$ transform under (2.9) as

$$
\begin{aligned}
N^{\prime i}\left(t^{\prime}, \mathbf{x}^{\prime}\right) & =N^{i}(t, \mathbf{x})+N^{j}(t, \mathbf{x}) \partial_{j} \zeta^{i}(t, \mathbf{x})-N^{i}(t, \mathbf{x}) \dot{f}(t)-\dot{\zeta}^{i}(t, \mathbf{x}), \\
N^{\prime}\left(t^{\prime}, \mathbf{x}^{\prime}\right) & =N(t, \mathbf{x})-N(t, \mathbf{x}) \dot{f}(t), \\
g_{i j}^{\prime}\left(t^{\prime}, \mathbf{x}^{\prime}\right) & =g_{i j}(t, \mathbf{x})-g_{i l}(t, \mathbf{x}) \partial_{j} \zeta^{l}(t, \mathbf{x})-\partial_{i} \zeta^{k}(t, \mathbf{x}) g_{k j}(t, \mathbf{x})
\end{aligned}
$$


and also

$$
a_{i}^{\prime}\left(t^{\prime}, \mathbf{x}^{\prime}\right)=a_{i}(t, \mathbf{x})-a_{j}(t, \mathbf{x}) \partial_{i} \xi^{j}(t, \mathbf{x}) .
$$

Following [9] we now perform the Hamiltonian analysis of theory defined by the action (2.3). We firstly determine the momenta conjugate to $N, N^{i}$ and $g_{i j}$ from (2.3)

$$
\begin{array}{ll}
p_{N}(\mathbf{x})=\frac{\delta S}{\delta \partial_{t} N(\mathbf{x})} \approx 0, & p_{i}(\mathbf{x})=\frac{\delta S}{\delta \partial_{t} N^{i}(\mathbf{x})} \approx 0, \\
p^{i j}(\mathbf{x})=\frac{\delta S}{\delta \partial_{t} g_{i j}(\mathbf{x})}=\sqrt{g} \mathcal{G}^{i j k l} K_{k l}(\mathbf{x}), &
\end{array}
$$

where the first line in (2.12) implies that $p_{N}(\mathbf{x}), p_{i}(\mathbf{x})$ are primary constraints of the theory. On the other hand with the help of the relation between $p^{i j}$ and $\partial_{t} g_{i j}$ given on the second line in (2.12) we easily find the corresponding Hamiltonian

$$
H=\int d^{D} \mathbf{x}\left(N\left(\mathcal{H}_{T}+\sqrt{g} V\right)+N^{i} \mathcal{H}_{i}+v^{i} p_{i}+v^{N} p_{N}\right),
$$

where $\mathcal{H}_{T}$ and $\mathcal{H}_{i}$ are given as

$$
\begin{aligned}
\mathcal{H}_{T} & =\frac{1}{\sqrt{g}} p^{i j} \mathcal{G}_{i j k l} p^{k l}+\sqrt{g} E^{i j} \mathcal{G}_{i j k l} E^{k l}, \\
\mathcal{H}_{i} & =-2 g_{i k} \nabla_{j} p^{k j},
\end{aligned}
$$

and where we included the primary constraints $p_{N}(\mathbf{x}) \approx 0, p_{i}(\mathbf{x}) \approx 0$. Note that $\mathcal{H}_{T}, \mathcal{H}_{i}$ take the same form as in Hořava-Lifshitz gravity.

As usual the preservation of the primary constraints $p_{i}(\mathbf{x}) \approx 0$ imply the secondary constraints

$$
\mathcal{H}_{i}(\mathbf{x}) \approx 0 .
$$

It is convenient to introduce the following slightly modified smeared form of this constraint

$$
\mathbf{T}_{S}(\xi)=\int d^{D} \mathbf{x}\left(\xi^{i}(\mathbf{x}) \mathcal{H}_{i}(\mathbf{x})+\xi^{i}(\mathbf{x}) \partial_{i} N(\mathbf{x}) p_{N}(\mathbf{x})\right)
$$

Note that the additional term in $\mathbf{T}_{S}$ is proportional to the primary constraint $p_{N}(\mathbf{x}) \approx 0$. The significance of this term will be clear when we calculate the Poisson bracket between $\mathbf{T}_{S}(\xi)$ and $a_{i}$.

Now we come to the most interesting property of the healthy extended Horava-Lifshitz gravity that is related to the requirement of the preservation of the primary constraint $\Theta_{1}(\mathbf{x}) \equiv p_{N}(\mathbf{x}) \approx 0$ during the time evolution of the system. Explicitly, the time evolution of this constraint is governed by following equation

$$
\partial_{t} \Theta_{1}(\mathbf{x})=\left\{\Theta_{1}(\mathbf{x}), H\right\}=-\mathcal{H}_{T}(\mathbf{x})-\sqrt{g} V++\frac{1}{N} \partial_{i}\left(N \sqrt{g} \frac{\delta V}{\delta a_{i}}\right)(\mathbf{x}) \equiv-\Theta_{2}(\mathbf{x}) \approx 0
$$

using

$$
\left\{p_{N}(\mathbf{x}), \int d^{D} \mathbf{y} N \sqrt{g} V(g, a)\right\}=-\sqrt{g} V(\mathbf{x})+\frac{1}{N} \partial_{i}\left(N \sqrt{g} \frac{\delta V}{\delta a_{i}}\right)(\mathbf{x})
$$


At this place we should stress one important point. Since $N$ is dynamical variable in healthy extended Hořava-Lifshitz theory it is natural to interpret the equation $\Theta_{2}(\mathbf{x}) \approx 0$ as the new secondary constraint between dynamical variables and that this constraint vanishes weakly. Only succeeding analysis of the consistency of this constraint with the time evolution of the system can determine whether this is the second class constraint that can be explicitly solved. Explicitly, the general analysis of the constraint systems implies that the total Hamiltonian is the sum of the original Hamiltonian and all constraints so that it takes the form

$$
H=\int d^{D} \mathbf{x}\left(N\left(\mathcal{H}_{T}+\sqrt{g} V\right)+N^{i}\left(\mathcal{H}_{i}+p_{N} \partial_{i} N\right)+v^{\alpha} \Theta_{\alpha}+v^{i} p_{i}\right)
$$

where $v^{\alpha}$ are Lagrange multipliers related to the constraints $\Theta_{\alpha}$. Observe that as opposite to the case of canonical gravity or standard Hořava-Lifshitz theory $N$ does not appear as Lagrange multiplier in the Hamiltonian (2.19).

As the next step we have to check the stability of the secondary constraints $\Theta_{2}(\mathbf{x}) \approx$ $0, \mathbf{T}_{S}(\xi) \approx 0$. In fact, using

$$
\begin{aligned}
\left\{\mathbf{T}_{S}(\xi), g_{i j}(\mathbf{x})\right\} & =-\xi^{k}(\mathbf{x}) \partial_{k} g_{i j}(\mathbf{x})-\partial_{i} \xi^{k}(\mathbf{x}) g_{k j}(\mathbf{x})-g_{i k}(\mathbf{x}) \partial_{j} \xi^{k}(\mathbf{x}) \\
\left\{\mathbf{T}_{S}(\xi), p^{i j}(\mathbf{x})\right\} & =-\partial_{k} p^{i j}(\mathbf{x}) \xi^{k}(\mathbf{x})-p^{i j}(\mathbf{x}) \partial_{k} \xi^{k}(\mathbf{x})+\partial_{k} \xi^{i}(\mathbf{x}) p^{k j}(\mathbf{x})+p^{i k}(\mathbf{x}) \partial_{k} \xi^{j}(\mathbf{x}) \\
\left\{\mathbf{T}_{S}(\xi), a_{i}(\mathbf{x})\right\} & =-\xi^{j}(\mathbf{x}) \partial_{j} a_{i}(\mathbf{x})-\partial_{i} \xi^{j}(\mathbf{x}) a_{j}(\mathbf{x})
\end{aligned}
$$

we easily find

$$
\begin{aligned}
\left\{\mathbf{T}_{S}(\xi), \mathcal{H}_{T}(\mathbf{x})\right\} & =-\xi^{k}(\mathbf{x}) \partial_{k} \mathcal{H}_{T}(\mathbf{x})-\mathcal{H}_{T}(\mathbf{x}) \partial_{k} \xi^{k}(\mathbf{x}), \\
\left\{\mathbf{T}_{S}(\xi), V(g(\mathbf{x}))\right\} & =-\partial_{i} V(\mathbf{x}) \xi^{i}(\mathbf{x}) .
\end{aligned}
$$

Collecting these results we find

$$
\left\{\mathbf{T}_{S}(\xi), \Theta_{\alpha}(\mathbf{x})\right\}=-\partial_{k} \Theta_{\alpha}(\mathbf{x}) \xi^{k}(\mathbf{x})-\Theta_{\alpha}(\mathbf{x}) \partial_{k} \xi^{k}(\mathbf{x}) .
$$

Then it is easy to see that the constraint $\mathbf{T}_{S}(\xi) \approx 0$ is preserved during the time evolution of the system since

$$
\partial_{t} \mathbf{T}_{S}(\xi)=\left\{\mathbf{T}_{S}(\xi), H\right\}=\int d^{D} \mathbf{x}\left(\partial_{k} v^{\alpha} \Theta_{\alpha} \xi^{k}\right)+\mathbf{T}_{S}\left(\xi^{i} \partial_{i} N^{k}-N^{i} \partial_{i} \xi^{k}\right) \approx 0
$$

using also the fact that

$$
\left\{\mathbf{T}_{S}(\xi), \mathbf{T}_{S}(\eta)\right\}=\mathbf{T}_{S}\left(\xi^{i} \partial_{i} \eta^{k}-\eta^{i} \partial_{i} \xi^{k}\right)
$$

As the next step we analyze the stability of constraints $\Theta_{1,2}$. To do this we calculate following Poisson bracket

$$
\left\{\Theta_{1}(\mathbf{x}), \Theta_{2}(\mathbf{y})\right\} \equiv \triangle_{12}(\mathbf{x}, \mathbf{y})=-\frac{1}{N} \partial_{y^{i}}\left(\sqrt{g} \frac{\delta^{2} V}{\delta a_{i}(\mathbf{y}) \delta a_{j}(\mathbf{y})}\left(a_{j}(\mathbf{y}) \delta(\mathbf{x}-\mathbf{y})-\partial_{y^{j}} \delta(\mathbf{x}-\mathbf{y})\right)\right) .
$$


Using this result we find that the time evolution of the constraint $\Theta_{1}(\mathbf{x})$ is equal to

$$
\begin{aligned}
\partial_{t} \Theta_{1}(\mathbf{x})= & \left\{\Theta_{1}(\mathbf{x}), H\right\}=\Theta_{1}(\mathbf{x})+\left\{\Theta_{1}(\mathbf{x}), \mathbf{T}_{S}\left(N^{i}\right)\right\}+ \\
& +\int d^{D} \mathbf{y} v_{2}(\mathbf{y})\left\{\Theta_{1}(\mathbf{x}), \Theta_{2}(\mathbf{y})\right\} \approx \int d^{D} \mathbf{y} v_{2} \triangle_{12}(\mathbf{x}, \mathbf{y}) .
\end{aligned}
$$

Clearly $\partial_{t} \Theta_{1} \approx 0$ for $v_{2}=0$. In the same way we determine the time evolution of the constraint $\Theta_{2}(\mathbf{x}) \approx 0$

$$
\partial_{t} \Theta_{2}(\mathbf{x})=\left\{\Theta_{2}(\mathbf{x}), H\right\} \approx \int d^{D} \mathbf{y}\left(N\left\{\Theta_{2}(\mathbf{x}), \mathcal{H}_{T}(\mathbf{y})+\sqrt{g} V(\mathbf{y})\right\}-v_{1} \triangle_{12}(\mathbf{y}, \mathbf{x})\right)=0
$$

using $v_{2}=0$ and also the fact that $\left\{\Theta_{2}(\mathbf{x}), \mathbf{T}_{S}\left(N^{i}\right)\right\} \approx 0$. We see from (2.27) that the requirement that $\partial_{t} \Theta_{2}(\mathbf{x})=0$ fixes $v_{1}$. As the result of this analysis we find following extended Hamiltonian

$$
H_{T}=H+\mathbf{T}_{S}\left(N^{i}\right)+\int d^{D} \mathbf{y} v^{i} p_{i}
$$

where

$$
H=\int d^{D} \mathbf{x}\left(N\left(\mathcal{H}_{T}+\sqrt{g} V\right)+v^{\alpha} \Theta_{\alpha}\right)=\int d^{D} \mathbf{x}\left(N\left(\mathcal{H}_{T}+\sqrt{g} V\right)+v_{1} \Theta_{1}\right)
$$

As then next step we explicitly solve $\Theta_{\alpha}$ in order to eliminate the canonical pair $p_{N}, N$ from the Hamiltonian. In this process we also replace the Poisson brackets between phase space variables $\left(g_{i j}, \pi^{i j}\right)$ defined on the reduced phase space with the Dirac brackets

$$
\begin{aligned}
\{F(g, p), G(g, p)\}_{D}= & \{F(g, p), G(g, p)\}- \\
& -\int d^{D} \mathbf{x} d^{D} \mathbf{y}\left\{F(q, p), \Theta_{\alpha}(\mathbf{x})\right\} \triangle^{\alpha \beta}(\mathbf{x}, \mathbf{y})\left\{\Theta_{\beta}(\mathbf{x}), G(p, q)\right\},
\end{aligned}
$$

where $\triangle^{\alpha \beta}(\mathbf{x}, \mathbf{y})$ is inverse of $\triangle_{\alpha \beta}(\mathbf{x}, \mathbf{y})$ in a sense

$$
\int d^{D} \mathbf{z} \triangle_{\alpha \beta}(\mathbf{x}, \mathbf{z}) \triangle^{\beta \gamma}(\mathbf{z}, \mathbf{y})=\delta_{\alpha}^{\gamma} \delta(\mathbf{x}-\mathbf{y}) .
$$

However due to the fact that the Poisson brackets between $g_{i j}, p^{i j}$ and $\Theta_{1}$ vanish we find that the Dirac brackets between canonical variables $g_{i j}, p^{i j}$ coincide with the Poisson brackets.

Let us now presume that the constraints $\Theta_{\alpha}=0$ can be explicitly solved. The solution of the first one $\Theta_{1}=0$ is clearly $p_{N}=0$. On the other hand it is very difficult to find the solution of the equation $\Theta_{2}=0$. We can only guess from the structure of the constraint $\Theta_{2}=0$ that $N$ has following functional dependence on $\mathcal{H}_{T}$ and $g_{i j}$

$$
N=N\left(\mathcal{H}_{T}, g\right)
$$

Then, using (2.32) in (2.29) we find that the Hamiltonian on the reduced phase space takes the form

$$
H_{T}=\int d^{D} \mathbf{x}\left[N\left(\mathcal{H}_{T}, g\right)\left(\mathcal{H}_{T}+\sqrt{g} V\left(N\left(\mathcal{H}_{T}, g\right)\right)\right)+v^{i} p_{i}\right]+\mathbf{T}_{S}\left(N^{i}\right)
$$


Note that the Hamiltonian (2.33) is not given as a linear combination of constraints which is a consequence of the fact that the Hamiltonian constraint is missing in the healthy extended Hořava-Lifshitz gravity. It is interesting to compare this result with the case of the General Relativity or Hořava-Lifshitz gravity with projectability condition where corresponding Hamiltonians are linear combinations of the first class constraints.

Remarkably, this fact also implies that the celebrated "problem of time" is absent in the healthy extended Hořava-Lifshitz gravity. As is well known the problem of time in General Relativity follows from the fact that General Relativity is a completely parameterized system. That is, there is no natural notion of time due to the diffeomorphism invariance of the theory and therefore the canonical Hamiltonian which generates time reparameterization vanishes. ${ }^{3}$ Explicitly, it is well known that the General Relativity Hamiltonian can be written as

$$
H^{G R}=\int d^{D} \mathbf{x}\left(N(\mathbf{x}) \mathcal{H}_{T}^{G R}(\mathbf{x})+N^{i}(\mathbf{x}) \mathcal{H}_{i}^{G R}(\mathbf{x})\right)
$$

where $\mathcal{H}_{T}^{G R}(\mathbf{x}) \approx 0, \mathcal{H}_{i}^{G R}(\mathbf{x}) \approx 0$ are generators of gauge transformations. Alternatively, we say that the General Relativity is complete constrained system defined as

$$
H_{T}^{G R}(N)=0, H_{S}^{G R}\left(N^{i}\right) \approx 0, \forall N, N^{i}
$$

where

$$
H_{T}^{G R}(N)=\int d^{D} \mathbf{x} N(\mathbf{x}) \mathcal{H}_{T}^{G R}(\mathbf{x}), \quad H_{S}^{G R}\left(N^{i}\right)=\int d^{D} \mathbf{x} N^{i}(\mathbf{x}) \mathcal{H}_{i}^{G R}(\mathbf{x}) .
$$

In fact, since the Hamiltonian is just a particular case of the gauge generator, the time evolution is just gauge, hence nothing happens. Time is frozen.

Let us now consider observable in General Relativity. By definition the observable is a phase space functional that weakly Poisson commutes with the smeared form of constraints [19]

$$
\left\{A(p, q), H_{T}^{G R}(N)\right\} \approx 0,\left\{A(p, q), H_{S}^{G R}\left(N^{i}\right)\right\} \approx 0
$$

or alternatively

$$
\left\{A(p, q), \mathcal{H}_{\alpha}^{G R}(\mathbf{x})\right\}=\int d^{D} \mathbf{x} \Lambda_{\alpha}^{\beta}(\mathbf{x}, \mathbf{y}) \mathcal{H}_{\beta}^{G R}(\mathbf{y}),
$$

where $\Lambda_{\alpha}^{\beta}$ generally depend on $p^{i j}, g_{i j}$ and where $\mathcal{H}_{\alpha}^{G R}=\left(\mathcal{H}_{T}^{G R}, \mathcal{H}_{i}^{G R}\right)$. From (2.34) we see that any observable is also an integral of motion that is another manifestation of the claim that the time is frozen.

Now we return to the construction of observables in healthy extended Hořava-Lifshitz gravity. By definition observable is a phase space function that weakly Poison commutes with generator of spatial diffeomorphism

$$
\left\{A(p, q), \mathbf{T}_{S}(\xi)\right\} \approx 0
$$

or alternatively

$$
\left\{A(p, q), \mathcal{H}_{i}(\mathbf{x})\right\}=\int d^{D} \mathbf{y} \Lambda_{i}^{j}(\mathbf{x}, \mathbf{y}) \mathcal{H}_{j}(\mathbf{y})
$$

\footnotetext{
${ }^{3}$ For detailed discussion of this issue see for example [20-24] where more references can be found.
} 
In other words, observable in this theory is any phase space functional that is invariant under spatial diffeomorphism. Clearly this requirement is much weaker than in General Relativity where the observable has to Poisson commute with Hamiltonian constraint as well.

Explicitly, let us discuss the time evolution of observable $A=A(p, g)$ that obeys (2.39). By definition

$$
\frac{d A}{d t}=\{A, H\} \approx \int d^{D} \mathbf{x}\left(\frac{\delta H}{\delta \mathcal{H}_{T}(\mathbf{x})}\left\{A, \mathcal{H}_{T}(\mathbf{x})\right\}+\frac{\delta H}{\delta g_{i j}(\mathbf{x})}\left\{A, g_{i j}(\mathbf{x})\right\}\right) .
$$

We see that the phase space functional that has following Poisson bracket with $\mathcal{H}_{T}$ and with $g_{i j}$

$$
\left\{A(p, q), \mathcal{H}_{T}(\mathbf{x})\right\}=\int d^{D} \mathbf{y} \Lambda^{i}(\mathbf{x}, \mathbf{y}) \mathcal{H}_{i}(\mathbf{y}), \quad\left\{A(p, q), g_{i j}(\mathbf{x})\right\}=\int d^{D} \mathbf{y} \Gamma_{i j}^{k}(\mathbf{x}, \mathbf{y}) \mathcal{H}_{k}(\mathbf{y})
$$

is integral of motion since

$$
\begin{aligned}
\frac{d A}{d t} & \approx \int d^{D} \mathbf{x} \int d^{D} \mathbf{y}\left(\frac{\delta H}{\delta \mathcal{H}_{T}(\mathbf{x})} \Lambda^{i}(\mathbf{x}, \mathbf{y}) \mathcal{H}_{i}(\mathbf{y})+\frac{\delta H}{\delta g_{i j}(\mathbf{x})} \Gamma_{i j}^{k}(\mathbf{x}, \mathbf{y}) \mathcal{H}_{k}(\mathbf{y})\right)= \\
& =\mathbf{T}_{S}\left(\int d^{D} \mathbf{x} \frac{\delta H}{\delta \mathcal{H}_{T}(\mathbf{x})} \Lambda^{i}(\mathbf{x}, \mathbf{y})+\int d^{D} \mathbf{x} \frac{\delta H}{\delta g_{k l}(\mathbf{x})} \Gamma_{k l}^{i}(\mathbf{x}, \mathbf{y})\right) \approx 0 .
\end{aligned}
$$

As a particular example of observable in healthy extended Hořava-Lifshitz gravity we consider the volume of spatial section

$$
\mathcal{V}=\int_{\Sigma} d^{D} \mathbf{x} \sqrt{g(\mathbf{x})}
$$

Then using

$$
\left\{g_{i j}(\mathbf{x}), \mathcal{H}_{T}(\mathbf{y})\right\}=2 \frac{1}{\sqrt{g}} \mathcal{G}_{i j k l} p^{k l}(\mathbf{x}) \delta(\mathbf{x}-\mathbf{y})
$$

we find

$$
\left\{\mathcal{V}, \mathcal{H}_{T}(\mathbf{x})\right\}=\frac{1}{1-\lambda D} g_{i j} p^{j i}(\mathbf{x})
$$

where we used

$$
g^{i j} \mathcal{G}_{i j k l}=\frac{1}{1-\lambda D} g_{k l} .
$$

Then it is easy to see that $\mathcal{V}$ depends on time since

$$
\frac{d \mathcal{V}}{d t}=\{\mathcal{V}, H\}=\frac{1}{1-\lambda D} \int d^{D} \mathbf{x} \frac{\delta H}{\delta \mathcal{H}_{T}(\mathbf{x})} g_{i j}(\mathbf{x}) p^{j i}(\mathbf{x}) \neq 0
$$

Now we compare this result with the situation in General Relativity. In the same way as above we find that

$$
\frac{d \mathcal{V}}{d t}=\frac{1}{1-D} \int d^{D} \mathbf{x} N(\mathbf{x}) g_{i j}(\mathbf{x}) p^{j i}(\mathbf{x})
$$

using the fact that $\lambda=1, \frac{\delta H^{G R}}{\delta \mathcal{H}_{T}^{G R}(\mathbf{x})}=N(\mathbf{x})$. However as opposite to the case of healthy extended Hořava-Lifshitz gravity $\mathcal{V}$ is not observable in the strick sense since it does not Poisson commute with the Hamiltonian constraint. 


\section{Batalin-Tyutin method for healthy extended Hořava-Lifshitz gravity}

In this section we show that the healthy extended Hořava-Lifshitz gravity can be formulated as the theory with the weakly vanishing first class constraints implementing the BatalinTyutin method [25]. The motivation for this analysis was the hope that turning the original healthy extended Hořava-Lifshitz gravity into the system with the first class constraint we would be able to find explicit form of the Hamiltonian and consequently more physical insight into the theory. Unfortunately we will see that the resulting theory is again very complicated with no explicit form of the phase space functionals derived.

To begin with we review the dynamical content of the healthy extended Horrava-Lifshitz theory. We have the dynamical fields $\left(g_{i j}, p^{i j}\right),\left(N^{i}, p_{i}\right),\left(N, p_{N}\right)$ together with following set of the first class constraints

$$
T_{a}=\left(\mathcal{H}_{i}(\mathbf{x}), p_{i}(\mathbf{x})\right) \approx 0 .
$$

and the second class constraints $\Theta_{\alpha}=0, \quad \alpha, \beta=1,2$

$$
\left\{\Theta_{\alpha}(\mathbf{x}), \Theta_{\beta}(\mathbf{y})\right\}=\triangle_{\alpha \beta}(\mathbf{x}, \mathbf{y}), \triangle_{\alpha \beta}(\mathbf{x}, \mathbf{y})=-\triangle_{\beta \alpha}(\mathbf{y}, \mathbf{x}) \text {. }
$$

Following [25] we introduce new fields $\Phi^{\alpha}$ with non-trivial Poisson brackets

$$
\left\{\Phi^{\alpha}(\mathbf{x}), \Phi^{\beta}(\mathbf{y})\right\}=\omega^{\alpha \beta}(\mathbf{x}, \mathbf{y}),
$$

where $\omega^{\alpha \beta}$ is antisymmetric field independent matrix so that

$$
\omega^{\alpha \beta}(\mathbf{x}, \mathbf{y})=-\omega^{\beta \alpha}(\mathbf{y}, \mathbf{x}) .
$$

Then the extended phase space is spanned by the variables $\left(g_{i j}, p^{i j}, N, p_{N}, N^{i}, p_{i}, \Phi^{\alpha}\right) \equiv$ $(p, q, \Phi)$ and our goal is to convert the second class constraints $\Theta_{\alpha}$ into the first class constraints that we denote as

$$
\mathcal{F}_{\alpha}=\mathcal{F}_{\alpha}(p, q, \Phi)=0
$$

By definition, the abelian conversion of the constraints is formulated as

$$
\left\{\mathcal{F}_{\alpha}(\mathbf{x}), \mathcal{F}_{\beta}(\mathbf{y})\right\}=0
$$

together with the boundary conditions

$$
\mathcal{F}_{\alpha}(p, q, 0)=\Theta_{\alpha}(p, q) .
$$

Then, following [25] we search the solution in the form

$$
\mathcal{F}_{\alpha}(p, q, \Phi)=\sum_{n=0}^{\infty} \mathcal{F}_{\alpha}^{(n)}, \quad \mathcal{F}_{\alpha}^{(n)} \sim \Phi^{n},
$$

where by definition

$$
\mathcal{F}_{\alpha}^{(0)}(p, q)=\Theta_{\alpha}(p, q)
$$


Inserting (3.8) into (3.6) we find

$$
\left\{\mathcal{F}_{\alpha}(\mathbf{x}), \mathcal{F}_{\beta}(\mathbf{y})\right\}=\sum_{n} \sum_{m}\left\{\mathcal{F}_{\alpha}^{(n)}(\mathbf{x}), \mathcal{F}_{\beta}^{(m)}(\mathbf{y})\right\}=0 .
$$

Now we demand that expressions of the same order in $\Phi^{\prime} s$ match. As was shown in [25] this requirement leads to the set of infinite recursive relations

$$
\begin{aligned}
& \left\{\mathcal{F}_{\alpha}^{(0)}(\mathbf{x}), \mathcal{F}_{\beta}^{(0)}(\mathbf{y})\right\}_{(p, q)}+\left\{\mathcal{F}_{\alpha}^{(1)}(\mathbf{x}), \mathcal{F}_{\beta}^{(1)}(\mathbf{y})\right\}_{(\Phi)}=0, \\
& \left\{\mathcal{F}_{[\alpha}^{(1)}(\mathbf{x}), \mathcal{F}_{\beta]}^{(n+1)}(\mathbf{y})\right\}_{(\Phi)}+B_{\alpha \beta}^{(n)}(\mathbf{x}, \mathbf{y})=0, n \leq 0,
\end{aligned}
$$

where

$$
\begin{aligned}
B_{\alpha \beta}^{(1)}(\mathbf{x}, \mathbf{y})= & \left\{\mathcal{F}_{[\alpha}^{(0)}(\mathbf{x}), \mathcal{F}_{\beta]}^{(1)}(\mathbf{y})\right\}_{(p, q)}, \\
B_{\alpha \beta}^{(n)}(\mathbf{x}, \mathbf{y})= & \frac{1}{2} B_{[\alpha \beta]}^{(n)}=\sum_{m=0}^{n}\left\{\mathcal{F}_{\alpha}^{(n-m)}(\mathbf{x}), \mathcal{F}_{\beta}^{(n)}(\mathbf{y})\right\}_{(p, q)}+ \\
& +\sum_{m=0}^{n-2}\left\{\mathcal{F}_{\alpha}^{(n-m)}(\mathbf{x}), \mathcal{F}_{\beta}^{m+2}(\mathbf{y})\right\}_{(\Phi)}, \quad n \geq 2
\end{aligned}
$$

and where $\{,\}_{(p, q)}$ denote the Poisson brackets with respect to $g_{i j}, p^{i j}, N^{i}, p_{i}, N, p_{N}$ and $\{,\}_{(\Phi)}$ denote the Poisson bracket with respect to $\Phi^{\prime} s$. Antisymmetrization in $\alpha, \beta$ indices is defined as

$$
K_{[\alpha \beta]}=K_{\alpha \beta}-K_{\beta \alpha} .
$$

Now we can straightforwardly construct the individual terms in expansion $\mathcal{F}_{\alpha}$. For example, $\mathcal{F}_{\alpha}^{(1)}$ is given as

$$
\mathcal{F}_{\alpha}^{(1)}(\mathbf{x})=\int d \mathbf{y} X_{\alpha \beta}(\mathbf{x}, \mathbf{y}) \Phi^{\beta}(\mathbf{y}),
$$

where $X_{\alpha \beta}$ obey the equation

$$
\int d^{D} \mathbf{z} d^{D} \mathbf{z}^{\prime} X_{\alpha \mu}(\mathbf{x}, \mathbf{z}) \omega^{\mu \nu}\left(\mathbf{z}, \mathbf{z}^{\prime}\right) X_{\nu \beta}\left(\mathbf{z}^{\prime}, \mathbf{y}\right)=-\triangle_{\alpha \beta}(\mathbf{x}, \mathbf{y}) .
$$

In order to obtain the complete series it is essential to introduce the matrix $\omega_{\alpha \beta}$ and $X^{\alpha \beta}$ that are inverse to $\omega_{\alpha \beta}$ and $X_{\alpha \beta}$ respectively

$$
\begin{aligned}
& \int d^{D} \mathbf{y} \omega^{\alpha \beta}(\mathbf{x}, \mathbf{y}) \omega_{\beta \gamma}(\mathbf{y}, \mathbf{z})=\delta_{\gamma}^{\alpha} \delta(\mathbf{x}-\mathbf{z}), \\
& \int d^{D} \mathbf{y} X^{\alpha \beta}(\mathbf{x}, \mathbf{y}) X_{\beta \gamma}(\mathbf{y}, \mathbf{z})=\delta_{\gamma}^{\alpha} \delta(\mathbf{x}-\mathbf{z}) .
\end{aligned}
$$

Then the particular solution of the inhomogeneous equation (3.12) is given by [25]

$$
\mathcal{F}_{\alpha}^{(n+1)}(\mathbf{x})=-\frac{1}{n+2} \int d^{D} \mathbf{z} d^{D} \mathbf{z}^{\prime} d^{D} \mathbf{z}^{\prime \prime} \Phi^{\mu}(\mathbf{z}) \omega_{\mu \nu}\left(\mathbf{z}, \mathbf{z}^{\prime}\right) X^{\nu \rho}\left(\mathbf{z}^{\prime}, \mathbf{z}^{\prime \prime}\right) B_{\rho \alpha}^{(n)}\left(\mathbf{z}^{\prime \prime}, \mathbf{x}\right) .
$$

The general solution of (3.12) can be derived by adding to it a term containing the solution of the homogeneous equation (3.12). However it was shown in [25] that arbitrariness in 
these solutions correspond to the canonical transformations in extended phase space. Then for the actual computational purpose it suffices to work with the solution (3.17).

Let us now consider functional $A(p, q)$ defined on the original phase space and denote its extension as $\tilde{A}(p, q, \Phi)$, where $\tilde{A}$ is such phase space functional that strongly Poisson commutes with constraints $\mathcal{F}_{\alpha}$

$$
\left\{\mathcal{F}_{\alpha}(\mathbf{x}), \tilde{A}\right\}=0
$$

and that obeys the boundary condition $\tilde{A}(p, q, 0)=A(p, q)$. Following [25] we write $\tilde{A}(p, q, \Phi)$ as power series in $\Phi$

$$
\tilde{A}(p, q, \Phi)=\sum_{n=0}^{\infty} \tilde{A}^{(n)}, \quad \tilde{A}^{(n)} \sim \Phi^{n} .
$$

Then it can be shown that the functionals $\tilde{A}^{(n)}$ are determined through following recursion rules

$$
\left\{\mathcal{F}_{\alpha}^{(1)}(\mathbf{x}), \tilde{A}^{(n+1)}\right\}_{(\Phi)}+G_{\alpha}^{(n)}(\mathbf{x})=0, n \leq 0
$$

where

$$
\begin{aligned}
G_{\alpha}^{(0)}(\mathbf{x})= & \left\{\mathcal{F}_{\alpha}^{(0)}, \tilde{A}^{(0)}\right\} \\
G_{\alpha}^{(1)}(\mathbf{x})= & \left\{\mathcal{F}_{\alpha}^{(1)}(\mathbf{x}), \tilde{A}^{(0)}\right\}+\left\{\mathcal{F}_{\alpha}^{(0)}(\mathbf{x}), \tilde{A}^{(1)}\right\}+\left\{\mathcal{F}_{\alpha}^{(2)}(\mathbf{x}), \tilde{A}^{(1)}\right\}_{(\Phi)}, \\
G_{\alpha}^{(n)}(\mathbf{x})= & \sum_{m=0}^{n}\left\{\mathcal{F}_{\alpha}^{(n-m)}(\mathbf{x}), \tilde{A}^{(m)}\right\}_{(p, q)}+\sum_{m=0}^{n-2}\left\{\mathcal{F}_{\alpha}^{(n-m)}(\mathbf{x}), \tilde{A}^{(m+2)}\right\}_{(\Phi)}+ \\
& +\left\{\mathcal{F}_{\alpha}^{(n+1)}(\mathbf{x}), \tilde{A}^{(1)}\right\}_{(\Phi)}, n \geq 2 .
\end{aligned}
$$

It can be shown that the particular solution of the inhomogeneous equation (3.20) takes the form

$$
\tilde{A}^{(n+1)}=-\frac{1}{n+1} \int d^{D} \mathbf{x} d^{D} \mathbf{y} d^{D} \mathbf{z} \Phi^{\mu}(\mathbf{x}) \omega_{\mu \nu}(\mathbf{x}, \mathbf{y}) X^{\nu \rho}(\mathbf{y}, \mathbf{z}) G_{\rho}^{(n)}(\mathbf{z}) .
$$

The most important example of the phase space functional is the Hamiltonian $H_{0}$ that in case of healthy extended Hořava-Lifshitz theory takes the form

$$
H_{0}=\int d^{D} \mathbf{x} N\left(\mathcal{H}_{T}+\sqrt{g} V\right)
$$

Following discussion given above we introduce the extended Hamiltonian $\tilde{H}$ defined on the extended phase space

$$
\tilde{H}=\tilde{H}(p, q, \Phi),
$$

where the strong involution is required

$$
\left\{\mathcal{F}_{\alpha}(\mathbf{x}), \tilde{H}\right\}=0 .
$$

Note also that this Hamiltonian is subject to the boundary condition

$$
\tilde{H}(p, q, 0)=H_{0}(p, q)
$$


As in (3.19) we express $\tilde{H}$ as

$$
\tilde{H}=\sum_{n=0}^{\infty} \tilde{H}^{(n)}, \tilde{H}^{(n)} \sim \Phi^{n}
$$

with the boundary condition

$$
\tilde{H}^{(0)}(p, q, \Phi)=\tilde{H}(p, q, 0)=H_{0}(p, q) .
$$

Again, $\tilde{H}^{(n)}$ can in principle be derived from the recursion relations. In particular, $\tilde{H}^{(1)}$ is equal to

$$
\tilde{H}^{(1)}=-\int d^{D} \mathbf{x} d^{D} \mathbf{y} d^{D} \mathbf{z} \Phi^{\mu}(\mathbf{x}) \omega_{\mu \nu}(\mathbf{x}, \mathbf{y}) X^{\nu \rho}(\mathbf{y}, \mathbf{z})\left\{\Theta_{\rho}(\mathbf{z}), H_{0}\right\} .
$$

Finally we should consider the extension of the original first class constraints $T_{a}$ given in (3.1). By definition these constraints have weakly vanishing Poisson brackets

$$
\left\{T_{a}(\mathbf{x}), T_{b}(\mathbf{y})\right\} \approx 0, \quad\left\{H_{0}, T_{a}(\mathbf{x})\right\} \approx 0, \quad\left\{\Theta_{\alpha}(\mathbf{x}), T_{b}(\mathbf{y})\right\} \approx 0 .
$$

As was shown in [25] their extension $\tilde{T}_{a}(p, q, \Phi), \tilde{T}_{a}(p, q, 0)=T_{a}(p, q)$ can be constructed in the same way as in case of general phase space functions. Moreover, it was also shown that they have following Poisson brackets

$$
\begin{aligned}
\left\{\tilde{T}_{a}(\mathbf{x}), \tilde{T}_{b}(\mathbf{y})\right\} & =\int d^{D} \mathbf{z} \tilde{U}_{a b}^{c}(\mathbf{x}, \mathbf{y}, \mathbf{z}) \tilde{T}_{c}(\mathbf{z})+\int d^{D} \mathbf{z} \tilde{I}_{a b}^{\alpha}(\mathbf{x}, \mathbf{y}, \mathbf{z}) \tilde{\Theta}_{\alpha}(\mathbf{z}), \\
\left\{\tilde{H}, \tilde{T}_{a}(\mathbf{x})\right\} & =\int d^{D} \mathbf{y} \tilde{V}_{a}^{b}(\mathbf{x}, \mathbf{y}) \tilde{T}_{b}(\mathbf{y})+\int d^{D} \mathbf{y} \tilde{K}_{a}^{\alpha}(\mathbf{x}, \mathbf{y}) \tilde{\Theta}_{\alpha}(\mathbf{y}),
\end{aligned}
$$

where $\tilde{\Theta}_{\alpha}(\mathbf{x}) \equiv \mathcal{F}_{\alpha}(\mathbf{x})$ and where $\tilde{U}, \tilde{K}, \tilde{V}$ are general phase space functions. Further, by definition of the abelian extension we have following strongly vanishing Poisson brackets

$$
\left\{\tilde{\Theta}_{\alpha}(\mathbf{x}), \tilde{T}_{a}(\mathbf{y})\right\}=\left\{\tilde{\Theta}_{a}(\mathbf{x}), \tilde{\Theta}_{b}(\mathbf{y})\right\}=\left\{\tilde{H}, \tilde{\Theta}_{a}(\mathbf{x})\right\}=0 .
$$

The outline of this analysis in the case of Hořava-Lifshitz gravity is following. We have original phase space variables $\left(g_{i j}, p^{i j}, N, p_{N}, N_{i}, p^{i}\right)$ together with additional scalar degrees of freedom $\left(\Phi^{1}, \Phi^{2}\right)$ with the phase space structure

$$
\left\{\Phi^{\alpha}(\mathbf{x}), \Phi^{\beta}(\mathbf{y})\right\}=\omega^{\alpha \beta}(\mathbf{x}, \mathbf{y}) .
$$

We also have the Hamiltonian $\tilde{H}$ together with the set of the first class constraints

$$
\mathcal{T}_{A}(\mathbf{x})=\left(\tilde{T}_{a}(\mathbf{x}), \tilde{\Theta}_{a}(\mathbf{x})\right) .
$$

The result of the Batalin-Tyutin construction is the healthy extended Hořava-Lifshitz gravity with the Hamiltonian

$$
\tilde{H}_{T}=\tilde{H}+\int d^{D} \mathbf{x} \lambda^{A}(\mathbf{x}) \mathcal{T}_{A}(\mathbf{x})
$$


where

$$
\begin{gathered}
\tilde{H}=H_{0}-\int d^{D} \mathbf{x} d^{D} \mathbf{y} d^{D} \mathbf{z} \Phi^{\mu}(\mathbf{x}) \omega_{\mu \nu}(\mathbf{x}, \mathbf{y}) X^{\nu \rho}(\mathbf{y}, \mathbf{z})\left\{\Theta_{\rho}(\mathbf{z}), H_{0}\right\}+\ldots \\
\tilde{\mathcal{H}}_{i}(\mathbf{x})=\mathcal{H}_{i}(\mathbf{x})-\int d^{D} \mathbf{y} d^{D} \mathbf{z} d^{D} \mathbf{z}^{\prime} \Phi^{\mu}(\mathbf{y}) \omega_{\mu \nu}(\mathbf{y}, \mathbf{z}) X^{\nu \rho}\left(\mathbf{z}, \mathbf{z}^{\prime}\right)\left\{\Theta_{\rho}\left(\mathbf{z}^{\prime}\right), \mathcal{H}_{i}(\mathbf{x})\right\}
\end{gathered}
$$

and where

$$
\begin{aligned}
& \tilde{\Theta}_{1}(\mathbf{x})=p_{N}(\mathbf{x})-\int d^{D} \mathbf{y} X_{1 \rho}(\mathbf{x}, \mathbf{y}) \Phi^{\rho}(\mathbf{y})+\ldots \\
& \tilde{\Theta}_{2}(\mathbf{x})=\mathcal{H}_{T}(\mathbf{x})+\sqrt{g} V(\mathbf{x})-\int d^{D} \mathbf{y} X_{2 \rho}(\mathbf{x}, \mathbf{y}) \Phi^{\rho}(\mathbf{y})+\ldots
\end{aligned}
$$

In summary we find the formulation of the healthy extended Hořava-Lifshitz gravity with the first class constraints only. Note that the Hamiltonian does not vanish on constraint surface that has important consequence for the time evolution of observable. Explicitly, let us consider observable that Poisson commute with all constraints

$$
\left\{\tilde{F}(p, q), \mathcal{T}_{A}(\mathbf{x})\right\} \approx 0
$$

Then its time evolution is governed by the equation

$$
\frac{d F}{d t}=\left\{\tilde{F}, \tilde{H}_{T}\right\} \approx\{\tilde{F}, \tilde{H}\}
$$

Further, as follows from (3.37) $p_{N}$ does not vanish in case of Batalin-Tyutin extension of the healthy extended Hořava-Lifshitz gravity. This fact implies that the corresponding Lagrangian contains time derivative of the lapse function in special way that is determined by the form of the first class constraint (3.37). Unfortunately as follows from the analysis given above it is very difficult to find corresponding Lagrangian and study this property in more details.

\section{Conclusion}

Let us conclude our paper. We studied the Hamiltonian formalism of the healthy extended Hořava-Lifshitz gravity. We found that the resulting theory seems to be well defined theory of gravity in the sense that governs the dynamics of metric components $g_{i j}$ and their conjugate momenta $p^{i j}$. Further, the Hamiltonian formalism of healthy extended Hořava-Lifshitz gravity shows rich structure of given theory with potentially interesting consequences. On the other hand we also discovered many puzzling properties related to given theory that certainly deserve further study. For example, it is very difficult to see how this theory is related to the Hamiltonian formulation of General Relativity. It is possible that such relation can be found between Batalin-Tyutin extended version of the healthy extended Hořava-Lifshitz gravity and General Relativity at least in some approximation. Further, it would be also extremely useful to find explicit dependence $N$ on $\mathcal{H}_{T}$ and $g$ at least approximately. We also mean that it would be interesting to include the 
boundary terms to the healthy extended Hořava-Lifshitz gravity and study their impact on the Hamiltonian formulation.

Despite all of these open problems we mean that the healthy extended Hořava-Lifshitz gravity is very interesting dynamical system in its own. Clearly further progress in its investigation would be desirable.

\section{Acknowledgments}

I would like to thank CERN PH-TH for generous hospitality and financial support during the course of this work. This work was also supported by the Czech Ministry of Education under Contract No. MSM 0021622409.

Open Access. This article is distributed under the terms of the Creative Commons Attribution Noncommercial License which permits any noncommercial use, distribution, and reproduction in any medium, provided the original author(s) and source are credited.

\section{References}

[1] P. Hořava, Quantum gravity at a Lifshitz point, Phys. Rev. D 79 (2009) 084008 [arXiv:0901.3775] [SPIRES].

[2] P. Hořava, Membranes at quantum criticality, JHEP 03 (2009) 020 [arXiv:0812.4287] [SPIRES].

[3] P. Hořava, Quantum criticality and Yang-Mills gauge theory, arXiv:0811.2217 [SPIRES].

[4] J. Kluson, Horava-Lifshitz f(R) gravity, JHEP 11 (2009) 078 [arXiv:0907.3566] [SPIRES].

[5] C. Charmousis, G. Niz, A. Padilla and P.M. Saffin, Strong coupling in Hořava gravity, JHEP 08 (2009) 070 [arXiv: 0905 .2579] [SPIRES].

[6] D. Blas, O. Pujolàs and S. Sibiryakov, On the extra mode and inconsistency of Hor̆ava gravity, JHEP 10 (2009) 029 [arXiv:0906.3046] [SPIRES].

[7] D. Blas, O. Pujolàs and S. Sibiryakov, Consistent extension of Hor̆ava gravity, Phys. Rev. Lett. 104 (2010) 181302 [arXiv:0909.3525] [SPIRES].

[8] D. Blas, O. Pujolàs and S. Sibiryakov, Comment on 'Strong coupling in extended Hořava-Lifshitz gravity', Phys. Lett. B 688 (2010) 350 [arXiv:0912.0550] [SPIRES].

[9] J. Kluson, Note about Hamiltonian formalism of modified $F(R)$ Hořava-Lifshitz gravities and their healthy extension, arXiv: 1002.4859 [SPIRES].

[10] M. Li and Y. Pang, A trouble with Horaava-Lifshitz gravity, JHEP 08 (2009) 015 [arXiv:0905.2751] [SPIRES].

[11] S. Weinfurtner, T.P. Sotiriou and M. Visser, Projectable Hor̆ava-Lifshitz gravity in a nutshell, J. Phys. Conf. Ser. 222 (2010) 012054 [arXiv: 1002. 0308] [SPIRES].

[12] M. Henneaux, A. Kleinschmidt and G.L. Gomez, A dynamical inconsistency of Horava gravity, Phys. Rev. D 81 (2010) 064002 [arXiv:0912.0399] [SPIRES].

[13] A. Papazoglou and T.P. Sotiriou, Strong coupling in extended Hor̆ava-Lifshitz gravity, Phys. Lett. B 685 (2010) 197 [arXiv:0911.1299] [SPIRES]. 
[14] J. Kluson, New models of $f(R)$ theories of gravity, Phys. Rev. D 81 (2010) 064028 [arXiv:0910.5852] [SPIRES].

[15] E. Gourgoulhon, 3+1 Formalism and bases of numerical relativity, gr-qc/0703035 [SPIRES].

[16] C.J. Isham, Canonical quantum gravity and the problem of time, gr-qc/9210011 [SPIRES].

[17] J. Govaerts, The quantum geometer's universe: particles, interactions and topology, hep-th/0207276 [SPIRES].

[18] J. Govaerts, Hamiltonian quantization and constrained dynamics, Leuven University Press, Leuven Belgium (1991) [SPIRES].

[19] C.G. Torre, Gravitational observables and local symmetries, Phys. Rev. D 48 (1993) 2373 [gr-qc/9306030] [SPIRES].

[20] T. Thiemann, Solving the problem of time in general relativity and cosmology with phantoms and $k$-essence, astro-ph/0607380 [SPIRES].

[21] B. Dittrich, Partial and complete observables for Hamiltonian constrained systems, Gen. Rel. Grav. 39 (2007) 1891 [gr-qc/0411013] [SPIRES].

[22] B. Dittrich, Partial and complete observables for canonical general relativity, Class. Quant. Grav. 23 (2006) 6155 [gr-qc/0507106] [SPIRES].

[23] J.D. Brown and K.V. Kuchar, Dust as a standard of space and time in canonical quantum gravity, Phys. Rev. D 51 (1995) 5600 [gr-qc/9409001] [SPIRES].

[24] J.M. Pons, D.C. Salisbury and K.A. Sundermeyer, Revisiting observables in generally covariant theories in the light of gauge fixing methods, Phys. Rev. D 80 (2009) 084015 [arXiv: 0905.4564] [SPIRES].

[25] I.A. Batalin and I.V. Tyutin, Existence theorem for the effective gauge algebra in the generalized canonical formalism with Abelian conversion of second class constraints, Int. J. Mod. Phys. A 6 (1991) 3255 [SPIRES].

[26] I. Kimpton and A. Padilla, Lessons from the decoupling limit of Hoŕava gravity, arXiv: 1003.5666 [SPIRES].

[27] K. Koyama and F. Arroja, Pathological behaviour of the scalar graviton in Horava-Lifshitz gravity, JHEP 03 (2010) 061 [arXiv:0910.1998] [SPIRES].

[28] J. Bellorín and A. Restuccia, On the consistency of the Horava theory, arXiv:1004.0055 [SPIRES].

[29] J.M. Pons and P. Talavera, Remarks on the consistency of minimal deviations from General Relativity, arXiv:1003.3811 [SPIRES].

[30] S. Carloni et al., Modified first-order Hořava-Lifshitz gravity: Hamiltonian analysis of the general theory and accelerating $F R W$ cosmology in power-law $F(R)$ model, arXiv:1003.3925 [SPIRES]. 